\title{
Dynamical Approach to the Cosmological Constant
}

\section{Citation}

Mukohyama, Shinji, and Lisa Randall. 2004. "Dynamical Approach to the Cosmological

Constant." Physical Review Letters 92 (21). https://doi.org/10.1103/physrevlett.92.211302.

\section{Permanent link}

http://nrs.harvard.edu/urn-3:HUL.InstRepos:41417289

\section{Terms of Use}

This article was downloaded from Harvard University's DASH repository, and is made available under the terms and conditions applicable to Other Posted Material, as set forth at http:// nrs.harvard.edu/urn-3:HUL.InstRepos:dash.current.terms-of-use\#LAA

\section{Share Your Story}

The Harvard community has made this article openly available.

Please share how this access benefits you. Submit a story.

Accessibility 


\title{
A Dynamical Approach to the Cosmological Constant
}

\author{
Shinji Mukohyama and Lisa Randall \\ Department of Physics, Harvard University \\ Cambridge, MA, 02138, USA
}

(October 29, 2018)

\begin{abstract}
We consider a dynamical approach to the cosmological constant. There is a scalar field with a potential whose minimum occurs at a generic, but negative, value for the vacuum energy, and it has a non-standard kinetic term whose coefficient diverges at zero curvature as well as the standard kinetic term. Because of the divergent coefficient of the kinetic term, the lowest energy state is never achieved. Instead, the cosmological constant automatically stalls at or near zero. The merit of this model is that it is stable under radiative corrections and leads to stable dynamics, despite the singular kinetic term. The model is not complete, however, in that some reheating is required. Nonetheless, our approach can at the very least reduce fine-tuning by 60 orders of magnitude or provide a new mechanism for sampling possible cosmological constants and implementing the anthropic principle.

[HUTP-03/A041]
\end{abstract}

The cosmological constant remains the biggest puzzle plaguing particle physics $[1,2]$. There is no known mechanism that guarantees zero, or nearly zero energy in a stable or metastable minimum energy configuration. Recently, the problem of the existence of small vacuum energy, whose value has to be about the same size as the energy in matter today, has further complicated the issue $[3-5]$. The apparent impossibility of addressing these problems has led to an increase in speculation for the necessity of the anthropic principle [6]. Before resorting to this, it is worthwhile to ask whether anything could possibly do what the cosmological constant data requires [2]. It seems likely that the correct way to interpret the tiny value of the cosmological constant is that conventional quantum field theory is not the whole story, so it is worth seeking acceptable modifications.

One might be tempted to consider non-standard potentials. However, those are never stable under radiative corrections. In this letter, we consider a new approach to the cosmological constant problem in which we try to avoid a fine-tuned potential, or one that would be unstable against radiative corrections. We propose that the apparent value of the cosmological constant is determined by dynamical considerations. The true value of the vacuum energy is not zero. But the dynamics is such that the true minimum is never attained, and the universe would settle down to a near zero energy state.

In this approach to the cosmological constant, we assume that inflation has already occurred, but ended when the vacuum energy was still large and positive. We propose scalar dynamics that decrease the cosmological vacuum energy in such a way that the scalar field stalls when the curvature becomes close to zero. The effective cos- mological constant is therefore zero or slightly positive.

We can think of two reasons this might happen. One possibility is that there is a scaling solution to scalar dynamics in which the kinetic and potential energies of a scalar field decrease in tandem, so that the field is slowed down when small vacuum energy is achieved. In our specific realizations, there was always an instability invalidating the models we tried.

The second possibility is that there is a feedback mechanism in which the equations for the scalar field depend on curvature in such a way that the field stalls at or near zero vacuum energy. This is the possibility that will be considered here.

Clearly, ordinary scalar field dynamics does not act the way we postulate. We assume the coefficient of a kinetic term diverges at zero curvature, so that the field gets frozen when the curvature approaches zero. Without such a singular term, the scalar field would overshoot and the energy would become negative. The singular kinetic term makes the scalar field stop at zero curvature, even without a fine-tuned potential.

The stability under radiative corrections is an important feature of our model. Radiative corrections will produce additional regular terms in the action, but the field will stall whether or not these are present.

One might also worry about additional singular potential terms being generated through radiative corrections but this does not happen. This can be seen explicitly using the Lagrangian below in Feynman diagrams or by a change of variables for which the kinetic term will be nonsingular, analogous to the Mukhanov choice of variable [7] in standard field theory cosmology.

The unconventional Lagrangian we consider is

$$
\begin{aligned}
I & =\int d^{4} x \sqrt{-g}\left[\frac{R}{2 \kappa^{2}}+\alpha R^{2}+L_{k i n}-V(\phi)\right], \\
L_{k i n} & =\frac{\kappa^{-4} K^{q}}{2 q f^{2 q-1}},
\end{aligned}
$$

where $f$ is a function of the Ricci scalar $R$ which vanishes at $R=0$ and behaves near $R=0$ as

$$
f(R) \sim\left(\kappa^{4} R^{2}\right)^{m}
$$

$\kappa$ is the Planck length, $\alpha$ and $q$ are constants, and $K \equiv-\kappa^{4} \partial^{\mu} \phi \partial_{\mu} \phi$. We do not give a reason why $f(R)$ vanishes at $R=0$. However, we shall show below that this choice of $f(R)$ is radiatively stable, leads to a stable dynamics and could help solve the cosmological constant problem. Our sign convention for the metric is 
$(-+++)$. We could make $f$ dependent of $\phi$, but such a dependence can be removed from the behavior of $L_{k i n}$ near $R=0$ by redefinition of $\phi$ without loss of generality. Note that $L_{k i n}$ above represents the term which is the most singular-looking at $R=0$ among many possible terms in the kinetic part and that we did not include less singular-looking terms since they are not important at low energy. We also omitted all other dynamical fields since, as we shall see below, the dynamics of $\phi$ is so slow that any dynamical fields other than $\phi$ will settle into their ground state before the universe approaches a sufficiently low energy state.

The goal is for the scalar field to stop rolling at or near zero vacuum energy without any fine-tuning of its potential. For this purpose, the minimum of the potential $V(\phi)$ should be negative so that $V(\phi)$ has a root. We can absorb any nonzero cosmological term into $V(\phi)$ without loss of generality as far as the minimum remains negative. We would like to stress again that all dynamical fields other than $\phi$ already settled into their ground state before the universe approaches a low energy state and that $V(\phi)$ includes the ground state energies of all such fields.

Notice there is no tuning in the potential. Any potential will work, so long as it has a minimum at negative energy. However, we do require a special form for the kinetic term. If all coefficients in the kinetic part are regular at zero curvature then it is evident that $\phi$ never stops there. Hence, we are forced to consider a singular kinetic term in which $f(R)$ vanishes at $R=0$. The only alternative would be to have $f$ depend on $\phi$ so that $f$ vanishes at the root of $V(\phi)$. However, this would not be stable under the addition of an extra vacuum energy to $V(\phi)$. Namely, $\phi$ does not know where to stop, whereas the curvature does.

We do not know a parent theory that will provide our Lagrangian as the low-energy effective theory. We treat this model as a purely phenomenological suggestion that might motivate further research into the possible parent theory, which is presumably not based entirely on conventional four-dimensional field theory.

We now show that this Lagrangian gives a feedback mechanism that makes the field stall at zero vacuum energy. We restrict this discussion to $q=1$. It is easy to see how the mechanism works for more general $q$.

In the flat Friedmann-Robertson-Walker background

$$
d s^{2}=-d t^{2}+a(t)^{2}\left(d x^{2}+d y^{2}+d z^{2}\right),
$$

the equation of motion for a homogeneous $\phi$ is

$$
\dot{\pi}+3 H \pi+V^{\prime}(\phi)=0, \quad \pi \equiv \dot{\phi} / f
$$

where $H=\dot{a} / a$, a dot denotes the time derivative, and $\pi$ is the momentum conjugate to $\phi$. As we shall see soon, $\phi$ evolves very slowly and, thus, $V$ can be approximated by a linear function near $V=0$ as

$$
V \simeq c \kappa^{-3}\left(\phi-\phi_{0}\right)
$$

where $c(=O(1))$ and $\phi_{0}$ are constants. Without any fine-tuning, the dimensionless constant $c$ should be of order unity. Hence, the asymptotic behavior of $\pi$ is

$$
\kappa^{2} \pi \sim-c \kappa^{-1} H^{-1}
$$

This follows when $\dot{H} / H^{2} \simeq$ const. $<0$ because the momentum $\pi$ under the influence of a constant force $\left(V^{\prime} \simeq\right.$ $\left.-c \kappa^{-3}\right)$ is asymptotically proportional to time and, thus, to $H^{-1}$. When $\dot{H} / H^{2} \simeq 0$, the above asymptotic form for $\pi$ is a consequence of the friction force $-3 H \pi$, which cancels the constant force when $3 H \pi+c \kappa^{-3}=0$. We shall soon see that $\dot{H} / H^{2} \simeq 0$ at low energy. If the kinetic term is small compared to the potential term then the Friedmann equation implies that

$$
3 H^{2} \simeq \kappa^{2} V
$$

and (6) can be rewritten as

$\kappa \partial_{t}\left(\kappa^{4} V\right) \sim c \kappa^{2} \pi f \sim-c^{2}(\kappa H)^{4 m-1} \sim-c^{2}\left(\kappa^{4} V\right)^{2 m-1 / 2}$.

Hence, we obtain

$$
\left(\kappa^{4} V\right)^{-2 m+3 / 2} \sim c^{2} \frac{t-t_{0}}{\kappa}
$$

where $t_{0}$ is a constant. If $-2 m+3 / 2<0$ then we achieve

$$
\kappa^{4} V \rightarrow+0 \quad(t / \kappa \rightarrow \infty)
$$

This means that $\phi$ stalls at $V=0$, where $V$ includes all contributions to the cosmological constant. Notice that the result (9) is independent of the values of $c$ and $\phi_{0}$. We also see that for a large $m(-2 m+3 / 2<-1 / 2), V$ approaches zero more slowly than $t^{-2}$ so that $\dot{H} / H^{2} \simeq 0$ at low energy, which was used above. We shall see later in this paper that stability requires (for $q=1$ )

$$
m>3 / 2 \text {. }
$$

With this condition, it is also easy to see that $V$ does not jump to a negative value by quantum fluctuation [11].

We have assumed that the kinetic energy is small compared to the potential energy. This assumption is easily justified. At low energy $(\kappa H \ll 1)$, if $m>1$ then

$$
L_{k i n}=\frac{f}{2} \pi^{2} \sim \frac{c^{2}}{\kappa^{4}}(\kappa H)^{4 m-2} \ll \frac{H^{2}}{\kappa^{2}} \sim V .
$$

We have achieved the vanishing cosmological constant in a way that is stable under radiative corrections and that has self-consistent, stable dynamics. However, although the cosmological constant approaches zero, it does so more slowly than matter or radiation so that without additional structure, the universe would be empty. It is not entirely clear that a dynamical model where this is not the case could be successful since it is this property that makes it possible for all fields other than $\phi$ to settle into their ground state before $\phi$ stalls 
at zero curvature so that the zero curvature really corresponds to the vanishing cosmological constant. Moreover, the large $m$ and, thus, the slow evolution of $\phi$ are required for stability. This does imply, however, that should this mechanism be responsible for a low cosmological constant, reheating would be required to thermally populate the universe after the cosmological constant has decreased to a small value. A couple of possibilities for the reheat process are:

(I) Low-energy inflation. One can consider an extra scalar field $\chi$ with mass $m_{\chi} \sim 10^{-3} \mathrm{eV}$ and a term like $-R \chi^{2}$. When $R \sim m_{\chi}^{2}$, a phase transition would occur (as in hybrid inflation [8]) and the universe would be reheated up to temperature $\sim T e V$. This phase transition happens when the energy stored in $\chi$ plus the energy stored in $\phi$ yields a Hubble constant of approximately $m_{\chi}$. The energy in $\chi$ will decrease during the phase transition; the energy after the phase transition must be very small. In this case the cosmological constant problem is reduced from $\left(M_{P l} / 10^{-3} \mathrm{eV}\right)^{4} \sim 10^{120}$ to $\left(T e V / 10^{-3} e V\right)^{4} \sim 10^{60}$. For smaller $m_{\chi}$, the reheat temperature would be lower and the tuning of the cosmological constant would presumably be smaller.

(II) Energy inflow from extra dimensions. For example, in a non-elastic scattering of branes, a part of the kinetic energy due to the relative motion can be converted to radiation on our brane without changing the brane tension and the cosmological constant. For this to work, branes should be sufficiently flat and parallel.

Although both these reheating mechanisms require some form of fine-tuning, one merit of the mechanism we have proposed is that after reheating, conventional cosmology with a vanishingly small cosmological constant would be restored. This is because the coefficient of the kinetic term is so large at low energy that the scalar field $\phi$ remains frozen after reheating. Namely,

$$
\left|\kappa^{2} \dot{\phi}\right| \sim(\kappa H)^{4 m} \times\left|\kappa^{2} \pi\right|
$$

can be made arbitrarily small at low energy $(\kappa H \ll 1)$ by considering a sufficiently large $m$ since $\pi$ always follows a regular equation of motion and, thus, evolves continuously. The stability condition (10) also requires a large $m$. Since $\phi$ rolls so slowly, our model in general predicts $w_{\phi} \equiv p_{\phi} / \rho_{\phi} \simeq-1$ today.

Any symmetry restoration or phase transitions that occurs after reheating would not pose a problem when $m$ is sufficiently large to freeze $\phi$. The reason is that the cosmological constant just before reheating is the value at zero temperature. The large $m$ ensures that $\Lambda_{\text {today }}$ is still positive and small since $\phi$ continues to be almost frozen all the way down to the present epoch including the time when the symmetry is restored and during the time the phase transition takes place.

If all else fails, although not our initial subjective, the existence of $\phi$ can at the very least provide a natural framework in which to implement the anthropic principle. If we assume eternal inflation, there would be many inflationary universe. In each universe, the cosmological constant is determined by how much $\phi$ has rolled when inflation ends. That in turn depends on the number of $e$-foldings that occurred before inflation stopped. In an eternal inflation scenario, different numbers of $e$-foldings would occur in different domains and therefore different $\phi$ values, and hence different values of the cosmological constant would occur in different regions.

One of the very interesting features of our model is that despite the singular-looking kinetic term, the scalar dynamics is stable for a broad range of parameters. Those parameter choices are (i) $q>1 / 2$; (ii) $\alpha>0$; and (iii) $2(m-1)>q /(2 q-1)$. In the $m \rightarrow \infty$ limit, the choice (2) may be replaced by $f(R) \sim \exp \left(-\kappa^{-4} R^{-2}\right)$ or similar functions. We would like to stress again that this condition is imposed only on the most singular-looking term among many possible terms in the kinetic part and, thus, adding any kinetic terms which are less singular-looking at $R=0$ does not change anything. Of course, adding a more singular-looking kinetic term just makes the condition more robust. The more singular a kinetic term looks, the more stable it is under radiative corrections.

Since $f$ is in the denominator and vanishes in the $\kappa^{2} R \rightarrow 0$ limit, the kinetic term threatens to be singular at low energy. Surprisingly, we shall see below that the singular-looking kinetic term with the large $m$ makes $\phi$ evolve slowly, that the numerator $K^{q}$ vanishes more quickly than the denominator and that the kinetic term is actually regular. The more singular a kinetic term looks, the more regular and stable the dynamics is.

Now let us briefly explain the reasons for the stability conditions (i)-(iii). (i) For the stability of inhomogeneous perturbations, it is necessary that the sound velocity squared $c_{s}^{2}=L_{k i n, K} /\left(2 K L_{k i n, K K}+L_{k i n, K}\right)$ is positive [9]. In our model this condition is reduced to $q>1 / 2$. (ii) The $R$-dependence of the kinetic term $L_{k i n}$ produces higher derivative corrections to Einstein equation which might destabilize gravity. As we shall explain below, the term $\alpha R^{2}$ can stabilize gravity at low energy if $\alpha$ is positive. (iii) For the term $\alpha R^{2}$ to control the stability, we need to make sure that the term $\alpha R^{2}$ is dominant over $L_{k i n}$ at low energy. As shown below, this is the case if and only if the condition (iii) is satisfied.

We can also show the essential, and somewhat surprising result, that the standard Friedmann equation is recovered at low energy, starting from the action (1). There are higher derivative corrections to the Einstein equation due to the $R$-dependence of $L_{k i n}$ and $\alpha R^{2}$.

Since there are higher-derivative terms, the stability of the system is a non-trivial question. In order to see the non-triviality, let us consider the Klein-Gordon equation $\left(\square-M^{2}\right) \varphi=0$ as a standard equation and add $\epsilon(\varphi) \square^{2} \varphi / M^{2}$ to the right hand side. One might expect that the standard equation should be recovered whenever $\epsilon \rightarrow 0$. Actually, this is not true. The standard equation is certainly recovered in the $\epsilon \rightarrow 0$ limit if $\epsilon>0$. On the other hand, if $\epsilon<0$ then the system has a tachyonic degree and is unstable. Moreover, if $\varphi$ crosses a root of $\epsilon$ 
then the system experiences a singularity ( $\square^{2} \varphi$ diverges) and the low energy effective theory cannot be trusted unless a miracle cancellation occurs.

In our system, specializing to the $q=1$ case again, from the estimate of $L_{k i n}$ in (11) with (10), $L_{k i n}$ is much smaller than $\alpha R^{2}\left(\sim H^{4}\right)$ at low energy if $\alpha \neq 0$. Hence, $\alpha R^{2}$ dominates the higher derivative corrections and controls the stability. For the theory $R / 2 \kappa^{2}+\alpha R^{2}$, the parameter $\alpha$ plays the role of $\epsilon$ above (including the sign) and it is known that the low energy dynamics is stable if and only if $\alpha \geq 0$ [10]. Here, stability means that as the universe expands, the system keeps away from unphysical spurious solutions and approaches the standard low energy evolution asymptotically. If we did not include the term $\alpha R^{2}$ then $L_{k i n}$ would make the quantity corresponding to $\epsilon$ above to be negative essentially because $f(R)$ is in the denominator. Therefore our system is stable and the standard Friedmann equation is recovered at low energy if and only if $\alpha>0$ and (10) (the condition (iii) above for a general $q>1 / 2$ ) are satisfied.

A possible source of instability which would disturb weak gravity in a Minkowski background could be an inhomogeneous fluctuation of $\phi$, which could possibly generate violent breakdown of linearized Einstein gravity. Quite surprisingly, this is not the case and the seemingly most dangerous part, the kinetic term, is not as dangerous as it looks. Essential to this conclusion is the constraint equation, which prevents $\phi$ from fluctuating freely and forces the denominator and the numerator to fluctuate in a strongly correlated way so that the contributions of the kinetic term to the equation of motion are regular and much smaller than those of the $\alpha R^{2}$ term [11]. Since contributions of the singular-looking kinetic term are small enough, the only possibly important correction to Einstein gravity is again due to the $\alpha R^{2}$ term. This tells us that the linearized gravity in our model should be identical to that in the theory $R / 2 \kappa^{2}+\alpha R^{2}$. Hence, we should be able to recover the linearized Einstein gravity in Minkowski background at distances longer than the length scale $l_{*}=\sqrt{\alpha} \kappa$ and at energies lower than $l_{*}^{-1}[12]$.

Before ending this letter, it is perhaps worth while stressing again that the way we have achieved the vanishing cosmological constant is stable under radiative corrections. Note that our essential assumption is that at least one coefficient in the kinetic part diverges at zero curvature. Although we did not give a reason why the divergence occurs at zero curvature, we showed that this assumption is stable under radiative corrections and leads to interesting dynamics. Adding extra terms to the action, irrespective of whether they are in the kinetic part or in the potential part, never spoils this assumption and, thus, the mechanism is stable under radiative corrections. The more singular a kinetic term looks, the more stable it is under radiative corrections.

Even more surprisingly, the seemingly most dangerous kinetic term does not lead to unstable dynamics. In fact, the more singular a kinetic term appears, the more regular and stable the dynamics is. In particular, we have shown not only that the cosmological constant vanishes but also that the standard Friedmann equation and the linearized Einstein gravity are recovered at low energy. This point is in itself of interest since it means there are new types of dynamics that can lead to the same type of weak gravity on a Minkowski background that we see today.

The feedback mechanism we have proposed can be considered as a way to protect a zero or small cosmological constant against radiative corrections. Hence, despite the necessity for late reheating and the lack of our knowledge about a parent theory, this may be the right starting point for thinking about the cosmological constant.

\section{ACKNOWLEDGMENTS}

We would like to thank N. Arkani-Hamed, J. Bloom, R. Bousso, H. C. Cheng, P. Creminelli, E. Flanagan, L. Motl and A. Strominger for useful comments. SM's work is supported by JSPS. This work was supported in part by NSF grant PHY-0201124 and PHY-9802709.

[1] S. Weinberg, Rev. Mod. Phys. 61, 1 (1989); P. J. E. Peebles and B. Ratra, Rev. Mod. Phys. 75, 559 (2003); T. Padmanabhan, Phys. Rept. 380, 235 (2003).

[2] E. Witten, hep-ph/0002297.

[3] S. Perlmutter, et al., Astrophys. J. 517, 565 (1999).

[4] B. P. Schmidt, et al., Astrophys. J. 507, 46 (1998).

[5] A. G. Riess et al., Astron. J. 116, 1009 (1998).

[6] For example, J. Garriga and A. Vilenkin, Phys. Rev. D67, 043503 (2003); R. Kallosh and A. Linde, Phys. Rev. D67, 023510 (2003); and references therein.

[7] V. F. Mukhanov, Sov. Phys. JETP 67, 1297 (1988).

[8] A. Linde, Particle physics and inflationary cosmology, Harwood (1990).

[9] J. Garriga and V. F. Mukhanov, Phys. Lett. B458, 219 (1999).

[10] V. Müller and H.-J. Schmidt, Gen. Rel. and Grav. 17, 769 (1985).

[11] S. Mukohyama, hep-th/0306208.

[12] K. S. Stelle, Gen. Rel. and Grav. 9, 353 (1978). 